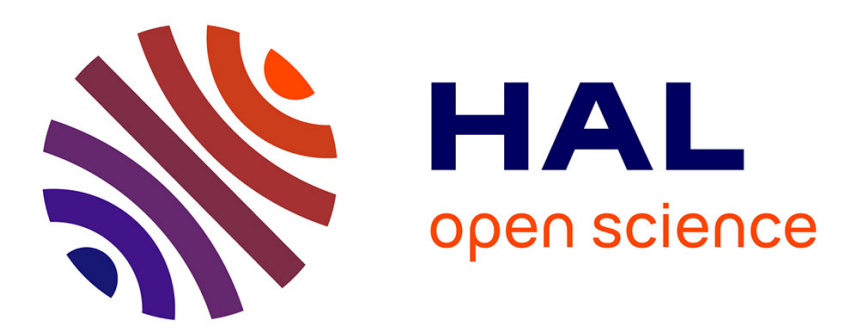

\title{
Characterization of polymorphic microsatellite loci in the terrestrial isopod Armadillidium vulgare
}

\author{
S. Verne, N. Puillandre, G. Brunet, N. Gouin, P. B. Samollow, J. D.
}

Anderson, F. Grandjean

\section{- To cite this version:}

S. Verne, N. Puillandre, G. Brunet, N. Gouin, P. B. Samollow, et al.. Characterization of polymorphic microsatellite loci in the terrestrial isopod Armadillidium vulgare. Molecular Ecology, 2006, 6, pp.328330. 10.1111/j.1471-8286.2006.01220.x . hal-00078079

\section{HAL Id: hal-00078079 \\ https://hal.science/hal-00078079}

Submitted on 2 Jun 2006

HAL is a multi-disciplinary open access archive for the deposit and dissemination of scientific research documents, whether they are published or not. The documents may come from teaching and research institutions in France or abroad, or from public or private research centers.
L'archive ouverte pluridisciplinaire HAL, est destinée au dépôt et à la diffusion de documents scientifiques de niveau recherche, publiés ou non, émanant des établissements d'enseignement et de recherche français ou étrangers, des laboratoires publics ou privés. 


\title{
PRIMER NOTE
}

\section{Characterization of polymorphic microsatellite loci in the terrestrial isopod Armadillidium vulgare}

\author{
SÉBASTIEN VERNE, ${ }^{*}$ NICOLAS PUILLANDRE, ${ }^{*}$ GÉRALDINE BRUNET,${ }^{*}$ NICOLAS GOUIN,+ \\ PAUL B. SAMOLLOW, $\ddagger$ JOEL D. ANDERSON† and FRÉDÉRIC GRANDJEAN* \\ *Laboratoire de Génétique et Biologie des Populations de Crustacés, UMR CNRS 6556, Université de Poitiers, 40 Avenue du Recteur \\ Pineau, 86022 Poitiers cedex, France, +Department of Genetics, Southwest Foundation for Biomedical Research, San Antonio, TX \\ 78245-0549, USA, †Department of Veterinary Integrative Bioscience, Texas AEM University, College Station, TX 77843-4458, USA
}

\begin{abstract}
The common pill bug, Armadillidium vulgare, is known to harbour two distinct strains of the feminizing proteobacteria Wolbachia. In order to study the effect of the presence of Wolbachia on the evolution of $A$. vulgare populations, we developed and characterized a set of nine polymorphic microsatellite loci from two microsatellite-enriched genomic libraries. We screened 48 individuals from three French populations and found high genetic variation. Locus-specific allelic diversity ranged from four to 28 and observed heterozygosity from 0.40 to 1.00 , which indicates that these markers can be used to conduct population genetic studies in A. vulgare.
\end{abstract}

Keywords: Armadillidium vulgare, Crustacea, Isopoda, microsatellites

Received 7 September 2005; revision accepted 20 October 2005

The common pill bug, Armadillidium vulgare (Crustacean, Isopod), is known to harbour two distantly related Wolbachia strains (Cordaux et al. 2004). Wolbachia are intracytoplasmic $\alpha$-proteobacteria that modify the reproduction of their hosts. Both strains induce feminization of genetic males into functional females in A. vulgare. Changes in host reproduction caused by Wolbachia can enhance the spread of the symbiont in infected populations (Werren \& O'Neill 1997) by increasing the frequency of infected cytoplasm at the expense of uninfected cytoplasm and by causing a decrease in mitochondrial DNA (mtDNA) polymorphism. However, the situation in A. vulgare is more complex because an unidentified non-Mendelian genetic element, called $f$, acts as a third feminizing sex-ratio distorter (Legrand \& Juchault 1984). Moreover, at least one 'resistance' gene inhibits vertical transmission of Wolbachia (Rigaud \& Juchault 1992) and one 'masculinizing' gene restores male phenotype in the presence of $f$ or Wolbachia (Rigaud \& Juchault 1993). As a consequence, Wolbachia infection does not spread. This can explain the presence of increased mtDNA polymorphism in infected $A$. vulgare populations compared to other infected isopod species (Rigaud

Correspondence: Sébastien Verne, Fax: +33 5494540 15; E-mail: sebastien.verne@free.fr et al. 1999). Although the host-parasite model A. vulgare/ Wolbachia has been studied for a long time, very little is known about the dispersal capabilities of $A$. vulgare and Wolbachia in the wild. To provide tools for examining the genetic structure of natural $A$. vulgare populations and the role of the feminizing factors on their genetic polymorphisms, we have isolated and characterized nine microsatellite loci for this species.

Isolation of microsatellite markers was achieved by the method of Hamilton et al. (1999). A detailed protocol is available at http://bioserver.georgetown.edu/faculty/ hamilton/. Briefly, total genomic DNA was extracted by a phenol-chloroform protocol (Kocher et al. 1989) from ovaries of one A. vulgare (Wolbachia non-infected) from a captive population. Twenty-five micrograms of genomic DNA was digested with restriction endonucleases HaeIII, RsaI, AluI and NheI (New England Biolabs) to generate DNA fragments within the 200-1000 bp range. These fragments were dephosphorylated using calf intestinal alkaline phosphatase and ligated to SNX linkers (SNX: 5'-CTAAGGCCTTGCTAGCAGAAGC-3'; SNX reverse: 5'pGCTTCTGCTAGCAAGGCCTTAGAAAA-3'). Two equal volumes of the linker-ligated genomic DNA solution $(10 \mu \mathrm{L})$ were then probed respectively with biotin-labelled $(\mathrm{AC})_{15}$ and $(\mathrm{AG})_{15}$ oligonucleotides. Library enrichment was achieved by a magnetic separation protocol employing 
streptavidin-coated polystyrene beads (Dynabeads M-280; Dynal, Inc.). The (AC)-enriched and the (AG)-enriched microsatellite libraries were constructed by cutting the enriched fragments with NheI, ligating the resulting products into dephosphorylated $\mathrm{XbaI}$-digested pBluescript plasmid, and transforming the ligated plasmids into Epicurian Coli XL2-Blue MRF Ultracompetent cells (Stratagene). Plasmid DNA was isolated using the QIAprep Spin Plasmid Kit (QIAGEN). Each library was screened with the same probe used for the bead enrichment protocol using a chemiluminescent detection kit (New England Biolabs). A total of 43 positive clones were sequenced using T3/T7 primers and BigDye termination mix (PerkinElmer Applied Biosystems) and resolved on an ABI 377 automated DNA analyser (Applied Biosystems). Sequences containing microsatellites were aligned using SEQUENCHER software (Gene Codes Corporation) to identify duplicate clones. Polymerase chain reaction (PCR) primer sets for amplification of the 43 cloned microsatellites were designed with PRIMER 3 software (http://frodo.wi.mit.edu/cgi-bin/ primer3/primer3_www.cgi).
PCRs were performed in a final volume of $12.5 \mu \mathrm{L}$ [1× Taq polymerase buffer (Promega: $500 \mathrm{~mm} \mathrm{KCl,} 100 \mathrm{~mm}$ Tris- $\mathrm{HCl} \mathrm{pH} 9.0,1 \%$ Triton $\mathrm{X}-100$ and $15 \mathrm{~mm} \mathrm{MgCl}_{2}$; except 1× TaKaRa buffer II for Av7: $100 \mathrm{~mm} \mathrm{KCl,} 100 \mathrm{~mm}$ Tris- $\mathrm{HCl}$ pH 8.0, 0.1 mм EDTA, 1 mm DTT, 0.5\% Tween 20, $0.5 \%$ Nonidet P-40, 50\% Glycerol), $1.2 \mathrm{mM} \mathrm{MgCl}_{2}, 60 \mathrm{M}$ of each dNTP, 5 pmol of each primer, $0.25 \mathrm{U}$ Taq polymerase (Promega, except LA Taq from TaKaRa for Av7) and $10 \mathrm{ng}$ of DNA template] using a Trio-Thermoblock (Biometra $\mathrm{GmBH})$. For each reaction, one primer of each pair was end labelled with one fluorescent phosphoramidite (TET, HEX or 6-FAM). The cycling programme consisted of an initial denaturing step of $5 \mathrm{~min}$ at $95^{\circ} \mathrm{C}, 35$ cycles of $30 \mathrm{~s}$ at $95^{\circ} \mathrm{C}$, $30 \mathrm{~s}$ at specific annealing temperature (Table 1), and $45 \mathrm{~s}$ at $72{ }^{\circ} \mathrm{C}$ followed by a final step of $10 \mathrm{~min}$ at $72{ }^{\circ} \mathrm{C}$. PCR products were separated along with the internal size standard GS500TAMRA on an ABI PRISM 310 automated sequencer. Product sizes were determined using the GENESCAN software (Applied Biosystems).

Nine polymorphic microsatellites were optimized. Table 1 presents details of allelic diversity and heterozygosity

Table 1 Polymorphic repeat microsatellite loci developed for the terrestrial isopod, Armadillidium vulgare

\begin{tabular}{|c|c|c|c|c|c|c|c|c|}
\hline $\begin{array}{l}\text { Locus (GenBank } \\
\text { Accession no.) }\end{array}$ & Primer sequences $\left(5^{\prime}-3^{\prime}\right)$ & $\begin{array}{l}\text { Repeat in } \\
\text { original clone }\end{array}$ & $\begin{array}{l}\text { Annealing } \\
\text { temp. }\left({ }^{\circ} \mathrm{C}\right)\end{array}$ & $\begin{array}{l}\text { No. of } \\
\text { alleles }\end{array}$ & $\begin{array}{l}\text { Size } \\
\text { range }(b p)\end{array}$ & Pop. & $H_{\mathrm{O}}$ & $H_{\mathrm{E}}$ \\
\hline $\begin{array}{l}\text { Av1 } \\
\text { DQ234304 }\end{array}$ & $\begin{array}{l}\mathrm{F}(1): \text { TGGAGTCAACTCACATTCTG } \\
\text { R: TGTCTGTAAAACTTGTGCTACG }\end{array}$ & $(\mathrm{CA})_{10}$ & 56 & 4 & $108-114$ & $\begin{array}{l}\text { Ens. } \\
\text { Poi. } \\
\text { Montp. }\end{array}$ & $\begin{array}{l}0.53 \\
0.53 \\
0.40\end{array}$ & $\begin{array}{l}0.68 \\
0.45 \\
0.58\end{array}$ \\
\hline $\begin{array}{l}\text { Av2 } \\
\text { DQ234305 }\end{array}$ & $\begin{array}{l}\text { F(1): TGAAGTTCGGGTGAATTGTG } \\
\text { R: ATACCATGACGTGTCGCAAG }\end{array}$ & $(\mathrm{CA})_{9}(\mathrm{CG})_{3}(\mathrm{CA})_{2}$ & 56 & 4 & $159-165$ & $\begin{array}{l}\text { Ens. } \\
\text { Poi. } \\
\text { Montp. }\end{array}$ & $\begin{array}{l}0.79 \\
0.89 \\
0.60\end{array}$ & $\begin{array}{l}0.69 \\
0.65 \\
0.75\end{array}$ \\
\hline $\begin{array}{l}\text { Av3 } \\
\text { DQ234306 }\end{array}$ & $\begin{array}{l}\mathrm{F}(2): \text { TGAGTCTCATTATAGTTTGGATGA } \\
\text { R: TCCTCTCTATACCCCATAATTTCA }\end{array}$ & $(\mathrm{CA})_{4}(\mathrm{CG})_{2}(\mathrm{CA})_{11}$ & 48 & 13 & $185-233$ & $\begin{array}{l}\text { Ens. } \\
\text { Poi. } \\
\text { Montp. }\end{array}$ & $\begin{array}{l}0.69 \\
0.65 \\
0.88\end{array}$ & $\begin{array}{l}0.83 \\
0.68 \\
0.89\end{array}$ \\
\hline $\begin{array}{l}\text { Av4 } \\
\text { DQ234307 }\end{array}$ & $\begin{array}{l}\mathrm{F}(1) \text { : CCGAACCTTTCGAAGGTATT } \\
\text { R: AAGGCACATAACATTTTCACAAA }\end{array}$ & $\begin{array}{l}(\mathrm{GT})_{4} \mathrm{AT}(\mathrm{GT})_{19} \\
(\mathrm{GA})_{4} \mathrm{AA}(\mathrm{GA})_{11}\end{array}$ & 56 & 16 & $198-242$ & $\begin{array}{l}\text { Ens. } \\
\text { Poi. } \\
\text { Montp. }\end{array}$ & $\begin{array}{l}0.45 \\
0.73 \\
0.90\end{array}$ & $\begin{array}{l}0.79 \\
0.82 \\
0.79\end{array}$ \\
\hline $\begin{array}{l}\text { Av5 } \\
\text { DQ234308 }\end{array}$ & $\begin{array}{l}\mathrm{F}(1) \text { : CGTGCGAAGTTCAGATTCTTT } \\
\text { R: GCGCGCTCGAGGATTTAC }\end{array}$ & $(\mathrm{CA})_{13}$ & 56 & 28 & $286-396$ & $\begin{array}{l}\text { Ens. } \\
\text { Poi. } \\
\text { Montp. }\end{array}$ & $\begin{array}{l}1.00 \\
0.95 \\
0.88\end{array}$ & $\begin{array}{l}0.94 \\
0.92 \\
0.94\end{array}$ \\
\hline $\begin{array}{l}\text { Av6 } \\
\text { DQ234309 }\end{array}$ & $\begin{array}{l}\mathrm{F}(1): \text { GGAATGAGGTCGTCGACTATG } \\
\text { R: GTCTTTCAAACGGGCACAAT }\end{array}$ & $(\mathrm{GT})_{8} \mathrm{AT}(\mathrm{GT})_{2}$ & 56 & 5 & $166-178$ & $\begin{array}{l}\text { Ens. } \\
\text { Poi. } \\
\text { Montp. }\end{array}$ & $\begin{array}{l}0.74 \\
0.63 \\
0.44\end{array}$ & $\begin{array}{l}0.56 \\
0.55 \\
0.53\end{array}$ \\
\hline $\begin{array}{l}\text { Av7 } \\
\text { DQ234310 }\end{array}$ & $\begin{array}{l}\text { F: TCATTTCCGCTTTCCTCATT } \\
\text { R(3): CCAACCCTGATTATGGTCTGAT }\end{array}$ & $(\mathrm{GT})_{2}(\mathrm{GC})_{7}(\mathrm{GT})_{11}$ & 50 & 8 & $229-251$ & $\begin{array}{l}\text { Ens. } \\
\text { Poi. } \\
\text { Montp. }\end{array}$ & $\begin{array}{l}0.50 \\
0.40 \\
0.60\end{array}$ & $\begin{array}{l}0.74 \\
0.63 \\
0.71\end{array}$ \\
\hline $\begin{array}{l}\text { Av8 } \\
\text { DQ234311 }\end{array}$ & $\begin{array}{l}\mathrm{F}(2) \text { : CAАCATCCTAATATTCAGTTCTCA } \\
\text { R: AGTTTACAGAATACGGCTGAGG }\end{array}$ & $(\mathrm{CA})_{32}$ & 48 & 15 & $152-192$ & $\begin{array}{l}\text { Ens. } \\
\text { Poi. } \\
\text { Montp. }\end{array}$ & $\begin{array}{l}0.74 \\
0.68 \\
0.70\end{array}$ & $\begin{array}{l}0.79 \\
0.84 \\
0.84\end{array}$ \\
\hline $\begin{array}{l}\text { Av9 } \\
\text { DQ234312 }\end{array}$ & $\begin{array}{l}\mathrm{F}(1): \text { TCTCGAAGAATTGCCTCACA } \\
\text { R: CGATGACTGGGACAATCTCA }\end{array}$ & $(\mathrm{CA})_{16}$ & 56 & 14 & $175-221$ & $\begin{array}{l}\text { Ens. } \\
\text { Poi. } \\
\text { Montp. }\end{array}$ & $\begin{array}{l}0.88 \\
0.84 \\
1.00\end{array}$ & $\begin{array}{l}0.84 \\
0.87 \\
0.89\end{array}$ \\
\hline
\end{tabular}

Label used and labelled primer: (1) 6-FAM. (2) HEX (3) TET.

Pop., population's name; Ens., Ensoulesse; Poi., Poitiers; Montp., Montpellier; $H_{\mathrm{O}}$, proportion of heterozygotes; $H_{\mathrm{E}^{\prime}}$, expected heterozygosity (calculated with GENEPOP version 3.3; Raymond \& Rousset 1995). Forty-eight individuals were amplified for each locus. 
Table 2 Allele sizes obtained by cross-species amplification using the primers developed for Armadillidium vulgare

\begin{tabular}{llllll}
\hline \multicolumn{7}{c}{ Species } & & & \\
\cline { 2 - 6 } Locus & Armadillidium nasatum & Armadillidium maculatum & Oniscus asellus & Porcellio scaber & Porcellionides pruinosus \\
\hline Av1 & - & - & - & - & - \\
Av2 & - & 145 & - & - & - \\
Av3 & 217 & $177-179$ & - & - & - \\
Av4 & - & - & - & - & - \\
Av5 & $374-386-410$ & $304-306$ & - & - & - \\
Av6 & 174 & 164 & - & - & - \\
Av7 & - & - & - & - & - \\
Av8 & $136-144$ & $122-124-130$ & - & - & - \\
Av9 & - & - & - & - \\
\hline
\end{tabular}

estimates at these nine loci in three widely separated French populations (Poitiers, Ensoulesse and Montpellier with sample sizes of 19,19 and 10 respectively). The number of alleles per locus ranged from four to 28 (average $=11.9$ ) and the observed heterozygosity from 0.40 to 1.00 (average $=0.75$ ), indicating a high level of polymorphism in A. vulgare populations. No significant genotypic linkage disequilibrium was found using Fisher's exact tests implemented in GENEPOP version 3.3 (Raymond \& Rousset 1995) after sequential Bonferroni correction $(\alpha=0.05, k=36)$. All the loci were tested separately in each population for Hardy-Weinberg equilibrium using GENEPOP version 3.3. No significant disequilibrium was detected after sequential Bonferroni correction $(\alpha=0.05, k=27)$. We also tested the utility of the primers in five other species representing three additional genera (Table 2). Two individuals from each species were tested at an annealing temperature of $48^{\circ} \mathrm{C}$. Some loci (Av3, Av5, Av6 and Av8) amplified successfully and exhibited polymorphisms in Armadillidium species, but none amplified in other species tested.

The polymorphisms observed at the nine microsatellite loci described herein should facilitate better understanding of genetic differentiation and structure among populations of A.vulgare and the evolutionary impact of sex-ratio distorters like Wolbachia on this species.

\section{Acknowledgements}

We thank Sylvie Patri for her technical assistance. We are grateful to Maryline Raimond, Catherine Debenest and Marie-Cécile Throuillé for assistance with collecting the woodlouse samples. The PhD work of Sébastien Verne is supported by a doctoral fellowship from the Région Poitou-Charentes.

\section{References}

Cordaux R, Michel-Salzat A, Frelon-Raimond M, Rigaud T, Bouchon D (2004) Evidence for a new feminizing Wolbachia strain in the isopod Armadillidium vulgare: evolutionary implications. Heredity, 93, 78-84.

Hamilton MB, Pincus EL, Di Fiore A, Fleischer RC (1999) Universal linker and ligation procedures for construction of genomic DNA libraries enriched for microsatellites. BioTechniques, 27, 500-507.

Kocher TD, Thomas WK, Meyer A, Edwards SV, Paabo S, Villablanca FX, Wilson AC (1989) Dynamics of mitochondrial DNA evolution in animals: amplification and sequencing with conserved primers. Proceedings of the National Academy of Sciences, USA, 86, 6196-6200.

Legrand JJ, Juchault P (1984) Nouvelles données sur le déterminisme génétique et épigénétique de la monogénie chez le crustacé isopode terrestre Armadillidium vulgare Latr. Genetics, Selection, Evolution, 16, 57-84.

Raymond M, Rousset F (1995) GENEPOP (version 1.2): population genetics software for exact tests and ecumenicism. Journal of Heredity, 86, 248-249.

Rigaud T, Bouchon D, Souty-Grosset C, Raimond R (1999) Mitochondrial DNA polymorphism, sex ratio distorters and population genetics in the isopod Armadillidium vulgare. Genetics, 152, 1669-1677.

Rigaud T, Juchault P (1992) Genetic control of the vertical transmission of a cytoplasmic sex factor in Armadillidium vulgare Latr. (Crustacea, Oniscidea). Heredity, 68, 47-52.

Rigaud T, Juchault P (1993) Conflict between feminizing sex ratio distorters and an autosomal masculinizing gene in the terrestrial isopod Armadillidium vulgare Latr. Genetics, 133, 247-252.

Werren JH, O'Neill S (1997) The evolution of heritable symbionts. In: Influential Passengers: Inherited Microorganisms and Arthropod Reproduction (eds O'Neill S, Hoffman A, Werren JH), pp. 125154. Oxford University Press, Oxford. 